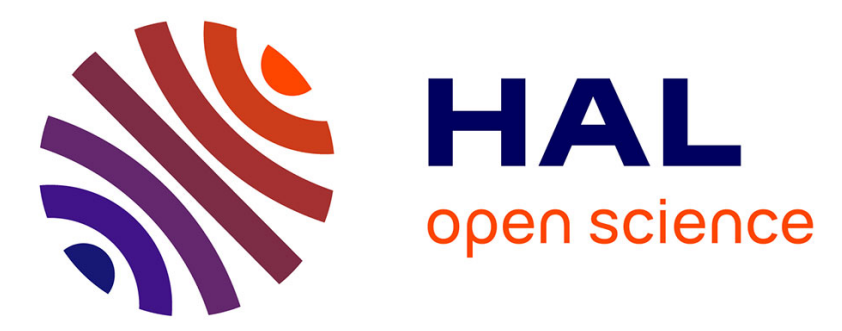

\title{
Very low frequency radio events with a reduced intensity observed by the low-altitude DEMETER spacecraft
}

J. Záhlava, F. Němec, O. Santolík, I. Kolmašová, Michel Parrot, C. J. Rodger

\section{To cite this version:}

J. Záhlava, F. Němec, O. Santolík, I. Kolmašová, Michel Parrot, et al.. Very low frequency radio events with a reduced intensity observed by the low-altitude DEMETER spacecraft. Journal of Geophysical Research Space Physics, 2015, 120, pp.9781-9794. 10.1002/2015JA021607 . insu-03576012

\section{HAL Id: insu-03576012 \\ https://hal-insu.archives-ouvertes.fr/insu-03576012}

Submitted on 15 Feb 2022

HAL is a multi-disciplinary open access archive for the deposit and dissemination of scientific research documents, whether they are published or not. The documents may come from teaching and research institutions in France or abroad, or from public or private research centers.
L'archive ouverte pluridisciplinaire HAL, est destinée au dépôt et à la diffusion de documents scientifiques de niveau recherche, publiés ou non, émanant des établissements d'enseignement et de recherche français ou étrangers, des laboratoires publics ou privés.

$$
\text { Copyright }
$$




\section{Journal of Geophysical Research: Space Physics}

\section{RESEARCH ARTICLE}

10.1002/2015JA021607

\section{Key Points:}

- Systematic study of unusual VLF radio events with a reduced intensity

- Geographic distribution of events shifted with respect to the lightning activity

- Model of event formation which is able to reproduce their basic properties

Correspondence to:

J. Záhlava,

jan.zahlava@centrum.cz

Citation:

Záhlava, J., F. Němec, O. Santolík, I. Kolmašová, M. Parrot, and C. J. Rodger (2015), Very low frequency radio events with a reduced intensity observed by the low-altitude DEMETER spacecraft, J. Geophys. Res. Space Physics, 120, 9781-9794, doi:10.1002/2015JA021607.

Received 22 JUN 2015 Accepted 15 OCT 2015

Accepted article online 19 OCT 2015 Published online 5 NOV 2015

(C)2015. American Geophysical Union. All Rights Reserved.

\section{Very low frequency radio events with a reduced intensity observed by the low-altitude DEMETER spacecraft}

\author{
J. Záhlava ${ }^{1}$, F. Němec' ${ }^{1}$, O. Santolík ${ }^{1,2}$, I. Kolmašová ${ }^{1,2}$, M. Parrot ${ }^{3}$, and C. J. Rodger ${ }^{4}$ \\ ${ }^{1}$ Faculty of Mathematics and Physics, Charles University in Prague, Prague, Czech Republic, ${ }^{2}$ Institute of Atmospheric \\ Physics, The Czech Academy of Sciences, Prague, Czech Republic, ${ }^{3}$ LPC2E/CNRS, Orléans, France, ${ }^{4}$ Department of Physics, \\ University of Otago, Dunedin, New Zealand
}

Abstract We present results of a systematic study of unusual very low frequency (VLF) radio events with a reduced intensity observed in the frequency-time spectrograms measured by the low-orbiting Detection of Electro-Magnetic Emissions Transmitted from Earthquake Regions (DEMETER) spacecraft. They occur exclusively on the nightside. During these events, the intensity of fractional hop whistlers at specific frequencies is significantly reduced. These frequencies are usually above about $3.4 \mathrm{kHz}$ (second Earth-ionosphere waveguide cutoff frequency), but about $20 \%$ of events extend down to about $1.7 \mathrm{kHz}$ (first Earth-ionosphere waveguide cutoff frequency). The frequencies of a reduced intensity vary smoothly with time. We have inspected 6.5 years of DEMETER data, and we identified in total 1601 such events. We present a simple model of the event formation based on the wave propagation in the Earth-ionosphere waveguide. We apply the model to two selected events, and we demonstrate that the model is able to reproduce both the minimum frequencies of the events and their approximate frequency-time shapes. The overall geographic distribution of the events is shifted by about $3000 \mathrm{~km}$ westward and slightly southward with respect to the areas with high long-term average lightning activity. We demonstrate that this shift is related to the specific DEMETER orbit, and we suggest its qualitative explanation by the east-west asymmetry of the wave propagation in the Earth-ionosphere waveguide.

\section{Introduction}

More than 6 years (2004-2010) of electromagnetic data measured by the low-altitude Detection of Electro-Magnetic Emissions Transmitted from Earthquake Regions (DEMETER) spacecraft contain a significant number of interesting and not yet well-understood phenomena. An extensive overview of these was recently given by Parrot et al. [2015]. We focus on the wave phenomenon shown in their Figure 12, i.e., very low frequency (VLF) wave events whose frequency-time spectrograms consist of many bands of reduced intensity that are evidently about $0.2 \mathrm{kHz}$ apart at $4 \mathrm{kHz}$ and almost $1 \mathrm{kHz}$ apart at $10 \mathrm{kHz}$ (Figure 1). Parrot et al. [2015] mentioned that these events are frequently observed during the nighttime and that they seem to be related to the thunderstorm activity. We provide a more detailed discussion of the event formation and properties in the present paper, along with a systematic analysis of the event occurrence. We also develop a simple model of the event formation, and we demonstrate its performance for two selected events.

Parrot et al. [2008] and Mazouz et al. [2011] reported curious events observed by the DEMETER spacecraft whose form in the frequency-time spectrograms resembled a V-shaped signature. They occurred at times when the satellite was passing above regions with a strong lightning activity (tens of lightning strokes per second), and they were shown to be related to the propagation of lightning-generated waves in the Earth-ionosphere waveguide prior to their leakage to the DEMETER altitudes (about $700 \mathrm{~km}$ ). Although a full-wave propagation code has been used to simulate detailed properties of these events [Parrot et al., 2008], the basic principle of their formation can be understood in terms of the waveguide mode theory [Budden, 1961].

Considering only transverse magnetic (TM) modes, which are excited by mostly vertical lightning currents, only the waves of the zero-order transverse magnetic (TM0) mode can propagate at frequencies below the first waveguide critical frequency (which is typically close to $1.7 \mathrm{kHz}$ during the nighttime). At frequencies above the waveguide critical frequency, an additional mode (TM1) can propagate. This usually results in a clearly identifiable boundary in frequency-time spectrograms measured by DEMETER, as the intensity of 


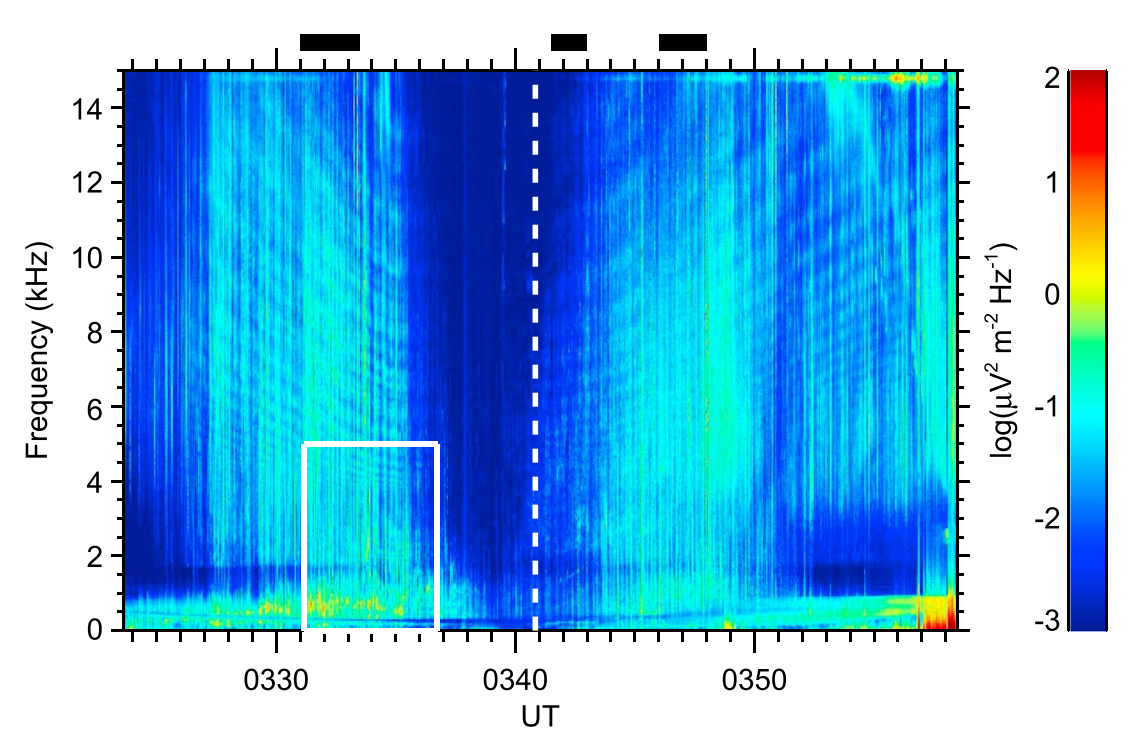

Figure 1. Frequency-time spectrogram of power spectral density of electric field fluctuations corresponding to one of the observed events. The frequencies of reduced intensity first decrease with time, then there is an apparent gap in the event, and, finally, the frequencies of reduced intensity increase with time. The data were measured on 7 February 2007. The white vertical dashed line at about 03:41 UT corresponds to the time when DEMETER crossed the geomagnetic equator. Black bars on the top mark the time intervals when the Burst mode was active and high-resolution data were available.

lightning-generated whistlers at frequencies higher than the waveguide critical frequency is much larger than their intensity below it [Toledo-Redondo et al., 2012]. At frequencies higher than the second waveguide critical frequency, there is an additional waveguide mode, TM2, etc. The individual waveguide modes propagate with different frequency-dependent phase speeds. When the resulting wave intensity is evaluated as the sum over all possible wave modes, they interfere with phase differences dependent upon the frequency and the length of the propagation path, which results in periodic variations of the intensity of the detected signal [e.g., Cummer, 2000]. We believe that the same mechanism is responsible for the formation of the events with a reduced intensity reported in this paper. However, in contrast to the formerly reported V-shaped events, these reduced intensity events are formed at considerably larger distances from the source storms. This results in their different spectral shape. Also, their occurrence rate is significantly higher, as there is no need for the spacecraft to pass almost over the storm for the events to form.

Our results show that lightning-generated signals are powerful enough to propagate over large distances in the Earth-ionosphere waveguide, exit it, and penetrate through the ionosphere far from the source thunderstorm. As a result of the interference of different waveguide modes of the propagating signals, the frequency spectrum of a series of resulting whistlers sometimes exhibits a characteristic strip-like pattern. This is potentially important for all studies of lightning effects on the Earth's electromagnetic environment.

The data that we have used in the present study are described in section 2. The obtained results are presented in section 3, and they are discussed in section 4. Finally, section 5 contains a brief summary.

\section{Data Set}

The DEMETER spacecraft operated between 2004 and 2010. It had a nearly circular orbit with an altitude of about $700 \mathrm{~km}$. The orbit was nearly polar and quasi Sun-synchronous so that DEMETER measurements were performed either shortly before the local noon (about 11:30 LT) or shortly before the local midnight (about 22:30 LT). The exact distribution of local times can be found in Figure 1 of Němec et al. [2010].

The DEMETER spacecraft was equipped with both electric field instruments [Berthelier et al., 2006] and magnetic field instruments [Parrot et al., 2006]. However, the magnetic field data contain a significant amount of spacecraft interferences, and only electric field data are thus used in the present study. In the VLF range (up to $20 \mathrm{kHz}$ ), onboard calculated frequency-time spectrograms of one electric field component are available. The measurements are continuous in time, but they are restricted to geomagnetic latitudes lower than about 
$65^{\circ}$. The frequency resolution of the spectrograms is $19.53 \mathrm{~Hz}$ (i.e., 1024 frequency channels between 0 and $20 \mathrm{kHz}$ ), and the time resolution is $2.048 \mathrm{~s}$. Moreover, waveforms of one electric field component with the sampling frequency of $40 \mathrm{kHz}$ are available during the Burst mode, which was active above locations of a special interest.

In addition to the electromagnetic wave data measured by the DEMETER spacecraft, we made use of the lightning data from the Lightning Imaging Sensor/Optical Transient Detector (LIS/OTD). The used 2.5 Degree Low Resolution Annual/Diurnal Climatology v2.2 is a $2.5^{\circ} \times 2.5^{\circ}$ gridded composite of climatological total (intracloud and cloud to ground) lightning bulk production as a function of local hour, expressed as a flash rate density (flashes $/ \mathrm{km}^{2} / \mathrm{yr}$ ). This is a combination of climatologies from the 5 year OTD (April 1995 to March 2000) and 8 year LIS (January 1998 to December 2005) missions. The best available detection efficiency corrections and instrument cross normalizations, as of the product generation date (6 January 2009), have been applied [Christian et al., 2003]. The data were recalculated to reflect only the local time intervals corresponding to the DEMETER spacecraft measurements, i.e., 8.7-11.7 h local time (daytime) and 20.7-23.7 h local time (nighttime) [Němec et al., 2010]. Moreover, the World Wide Lightning Locations Network (WWLLN) data were used for selected time intervals. These provide us with locations and times of lightning strokes which occurred all over the world during the selected time intervals [Lay et al., 2004; Rodger et al., 2004].

An example frequency-time spectrogram of power spectral density of electric field fluctuations corresponding to one of the observed reduced intensity events is shown in Figure 1. The data were measured during a nighttime half orbit on 7 February 2007. The frequencies of reduced intensity observed above about $4 \mathrm{kHz}$ first decrease with time, then there is an apparent about 7 min long gap in the event, and finally, the frequencies of reduced intensity increase with time. The white vertical dashed line at about 03:41 UT corresponds to the time when DEMETER crossed the geomagnetic equator. The apparent gap in the event, when the intensity of the detected emissions significantly decreases, occurs at about the time of the geomagnetic equator crossing. Taking into account (as we demonstrate further) that the observed emissions are lightning-generated whistlers [Helliwell, 1965], the decrease of the emission intensity can be likely explained by the lower efficiency of the wave penetration through the ionosphere in this region [e.g., Němec et al., 2008]. Black bars at the top of Figure 1 mark the time intervals when the Burst mode was active and high-resolution data were available.

A noteworthy feature of the example reduced intensity event is that the frequency spacing between the frequencies of reduced intensity increases with the frequency, which turns out to be the case for all identified events. At higher frequencies, the frequency spacing becomes rather large and the intensity of the emissions quite low so that it is difficult to properly determine the upper frequency boundary of the event. For this particular event we can estimate the upper frequency to be equal to about $14 \mathrm{kHz}$. One can also identify the waveguide critical frequency of about $1.7 \mathrm{kHz}$, where the intensity of the observed emissions has a rather sharp intensity cutoff [Toledo-Redondo et al., 2012], indicating that the emissions are indeed affected by the propagation in the Earth-ionosphere waveguide.

Curiously, the reduced intensity event from Figure 1 is accompanied by additional bands of reduced intensity between about 1.8 and $2.5 \mathrm{kHz}$, which occur shortly before the time of the apparent gap in the emissions (around 03:35 UT). As this additional reduced intensity event is too weak to be seen properly in Figure 1, a zoomed frequency-time spectrogram corresponding to the interval marked by the white rectangle is shown in Figure 2a. The additional reduced intensity event can be seen in the area marked by the white rectangle in Figure 2a. It starts just above the waveguide critical frequency at about $1.7 \mathrm{kHz}$. The main reduced intensity event then starts at frequencies of about $3.5 \mathrm{kHz}$, which approximately corresponds to the second waveguide critical frequency.

Geographic locations of the DEMETER spacecraft during the time interval analyzed in Figure 1 are shown in Figure $2 \mathrm{~b}$ by a thin curve. The part of the DEMETER orbit corresponding to the zoomed interval from Figure 2a is shown by the solid red rectangle. The parts of the spacecraft orbit where the Burst mode was active are shown by thick black curves. We note that DEMETER moved from the south to the north (as is the case for all nighttime half orbits). We selected the first Burst mode interval from Figure 1 to plot a detailed frequency-time spectrogram of this part of the event, in order to demonstrate that it is really formed by lightning-generated whistlers. A detailed frequency-time spectrogram of power spectral density of electric field fluctuations corresponding to the whole Burst mode interval is shown in Figure 3a. Figure $3 \mathrm{~b}$ then shows a very detailed frequency-time spectrogram corresponding to a $5 \mathrm{~s}$ long time interval marked by the black bar on the top of Figure 3a. 

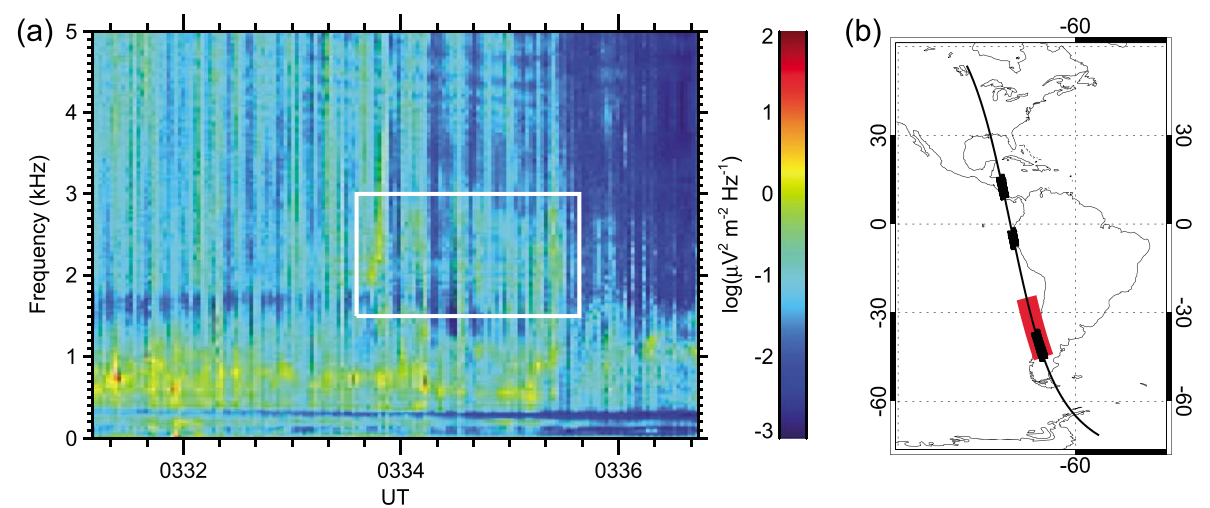

Figure 2. (a) Zoomed frequency-time spectrogram of power spectral density of electric field fluctuations corresponding to the interval marked by the white rectangle in Figure 1. The white rectangle marks an additional faint reduced intensity event at lower frequencies. (b) Map showing the DEMETER locations during the time interval analyzed in Figure 1 (thin curve). The part of the DEMETER orbit corresponding to the zoomed interval from Figure 2a is shown by the solid red rectangle. Parts of the DEMETER orbit with the active Burst mode are shown by the thick black curves. Note that DEMETER moved from the south to the north.

It can be seen that the emissions forming the event consist of many consecutive lightning-generated whistlers, which are significantly attenuated at some specific frequencies. This clearly demonstrates that the events are really formed due to a selective reduction of the wave intensity at some specific frequencies rather than due to a frequency-dependent source. The dispersion of the whistlers is rather low, meaning that they were dispersed only during their passage from the Earth-ionosphere waveguide through the ionosphere (fractional hop $0+$ whistlers). The frequencies of reduced intensity are nearly constant on time/spatial scales analyzed in Figure 3, but they change considerably on time/spatial scales analyzed in Figure 1.

We note that using a single spacecraft, one cannot in general directly distinguish between spatial and temporal variations of analyzed phenomena. Nevertheless, as we demonstrate further, in the case of the reduced intensity events the variations are spatial (i.e., due to the spacecraft movement) rather than temporal. Essentially, we believe that many lightning strokes are occurring at about the same place (source storm) is needed to form a reduced intensity event. The shape of the reduced intensity event is then related to the spacecraft movement with respect to the source storm, i.e., to the varying distance across which the lightning-generated emissions have to propagate in the Earth-ionosphere waveguide.

We visually inspected all VLF Survey mode data measured by the DEMETER spacecraft during the whole duration of its mission (2004-2010) for the presence of reduced intensity events. This inspection was done using spectrograms of whole DEMETER half orbits, which individually corresponded to about $35 \mathrm{~min}$ of data. The frequency range was from 0 to $20 \mathrm{kHz}$, the same for all the spectrograms. The power spectral density scale was also fixed, ranging from $10^{-3}$ to $10^{2} \mu \mathrm{V}^{2} \mathrm{~m}^{-2} \mathrm{~Hz}^{-1}$. The events are found to occur exclusively during the
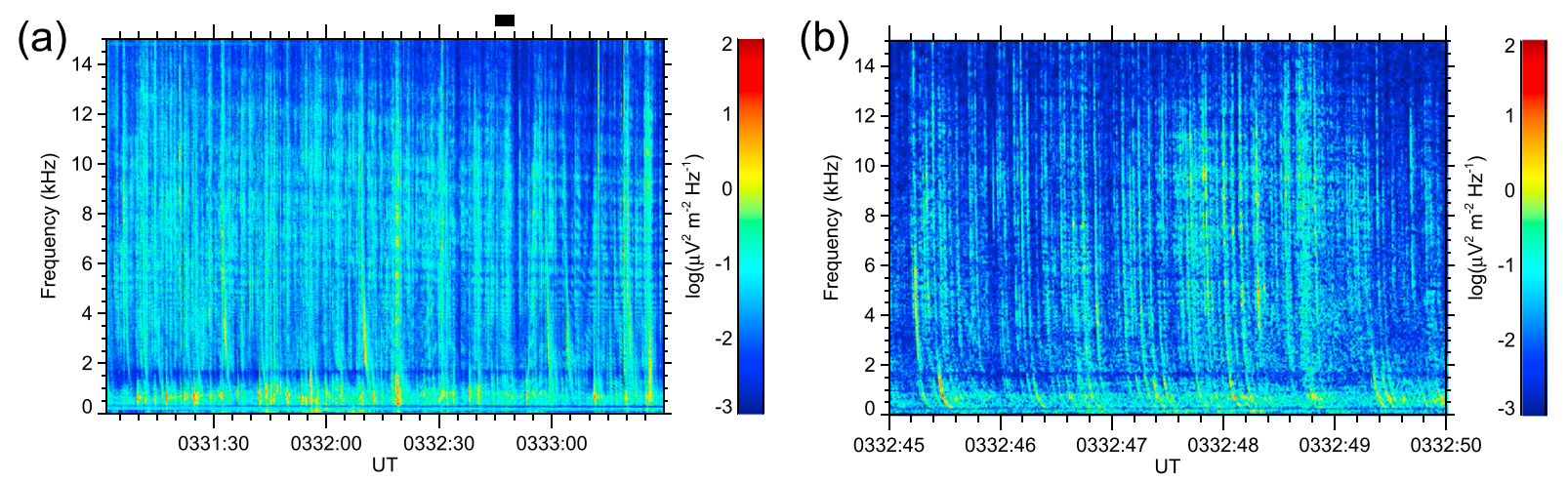

Figure 3. (a) Detailed frequency-time spectrogram of power spectral density of electric field fluctuations corresponding to the first Burst mode interval from Figure 1. (b) Detailed frequency-time spectrogram corresponding to a $5 \mathrm{~s}$ long time interval marked by the black bar on the top of Figure $3 \mathrm{a}$. 


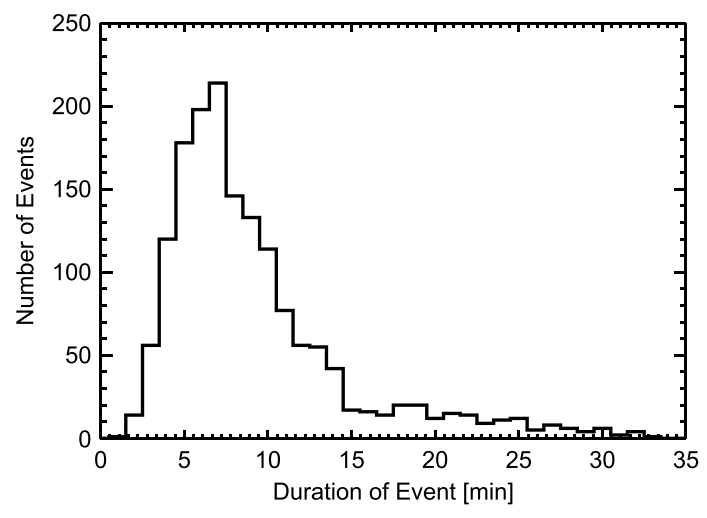

Figure 4. Histogram of the observed event durations.

nighttime half orbits. Altogether, 1601 reduced intensity events were identified in 28,670 nighttime spectrograms, thus occurring in about $6 \%$ of all nighttime orbits. Among these, 312 events were accompanied by additional reduced intensity events at lower frequencies, as described above for the example case in Figure 1 at about 03:35 UT. For each event, we have recorded its minimum and maximum frequencies and its beginning and ending times. Moreover, the times when the system of reduced intensity curves reached their minimum frequency were recorded for each event. These parameters were used as a starting point for further analysis.

\section{Results}

A histogram of event durations as they were observed by DEMETER is shown in Figure 4. It can be seen that the events typically last from about 5 to $10 \mathrm{~min}$. However, some events may last for as long as 30 min, i.e., principally, a whole DEMETER half orbit. Taking into account the spacecraft orbit, one can try to estimate the corresponding spatial dimensions. Namely, DEMETER moves by about 3.7 latitudinal degrees per minute, corresponding to some $400 \mathrm{~km} / \mathrm{min}$. The typical dimensions of the observed reduced intensity events are thus between 2000 and $4000 \mathrm{~km}$.

The frequencies of the reduced intensity events are analyzed in Figure 5. The frequency resolution of our analysis is $0.5 \mathrm{kHz}$. Unfortunately, it is not possible to achieve a better frequency resolution, as it is often difficult to determine the exact minimum and maximum frequencies of the events. Figure 5a shows a histogram of the minimum frequencies of the events. It can be seen that the minimum frequencies of the events range from 3 to $4 \mathrm{kHz}$. Taking into account the limited frequency resolution, this range of frequencies is consistent with the second critical frequency of the Earth-ionosphere waveguide, which is equal to about $3.4 \mathrm{kHz}$ during the night. A histogram of maximum frequencies of the events is shown in Figure $5 \mathrm{~b}$. The distribution is rather broad, peaking at about $9 \mathrm{kHz}$. We note that the broadness of the distribution is at least partially caused by the typically fuzzy upper boundary of the events and the related inaccuracy of its identification. Finally, Figure 5c shows a histogram of minimum frequencies of lower frequency reduced intensity events, which sometimes occur along with the normal reduced intensity events. It can be seen that the identified lower frequencies of these events are either $1.5 \mathrm{kHz}$ or $2.0 \mathrm{kHz}$; i.e., they are consistent with the first critical frequency of the Earth-ionosphere waveguide, which is equal to about $1.7 \mathrm{kHz}$ during the night.

The geographic locations of all 1601 reduced intensity events are shown in Figure 6a. For each event, the location is determined by the location of the DEMETER spacecraft at the time of the minimum frequency of the system of lines. As we show further, this location corresponds to the spacecraft location at the time when it was closest to the source storm. The number of events in each $2.5^{\circ} \times 2.5^{\circ}$ bin is color coded according to the scale on the top. Three areas of enhanced event occurrence can be identified. One of these areas is located in the equatorial region to the west of central Africa. Two other areas of increased event occurrence are located to the west of America, one in the Northern Hemisphere and one in the Southern Hemisphere. The distribution of geographic locations of the additional reduced intensity events at lower frequencies which sometimes occur along with the normal reduced intensity events are very similar to the locations of the normal reduced intensity events shown in Figure 6a. Taking into account that the events are due to the intensity modulation of lightning-generated whistlers, it is interesting to compare event and lightning locations. Although the event formation is related to an intense localized thunderstorm rather than global lightning activity, it is instruc- 

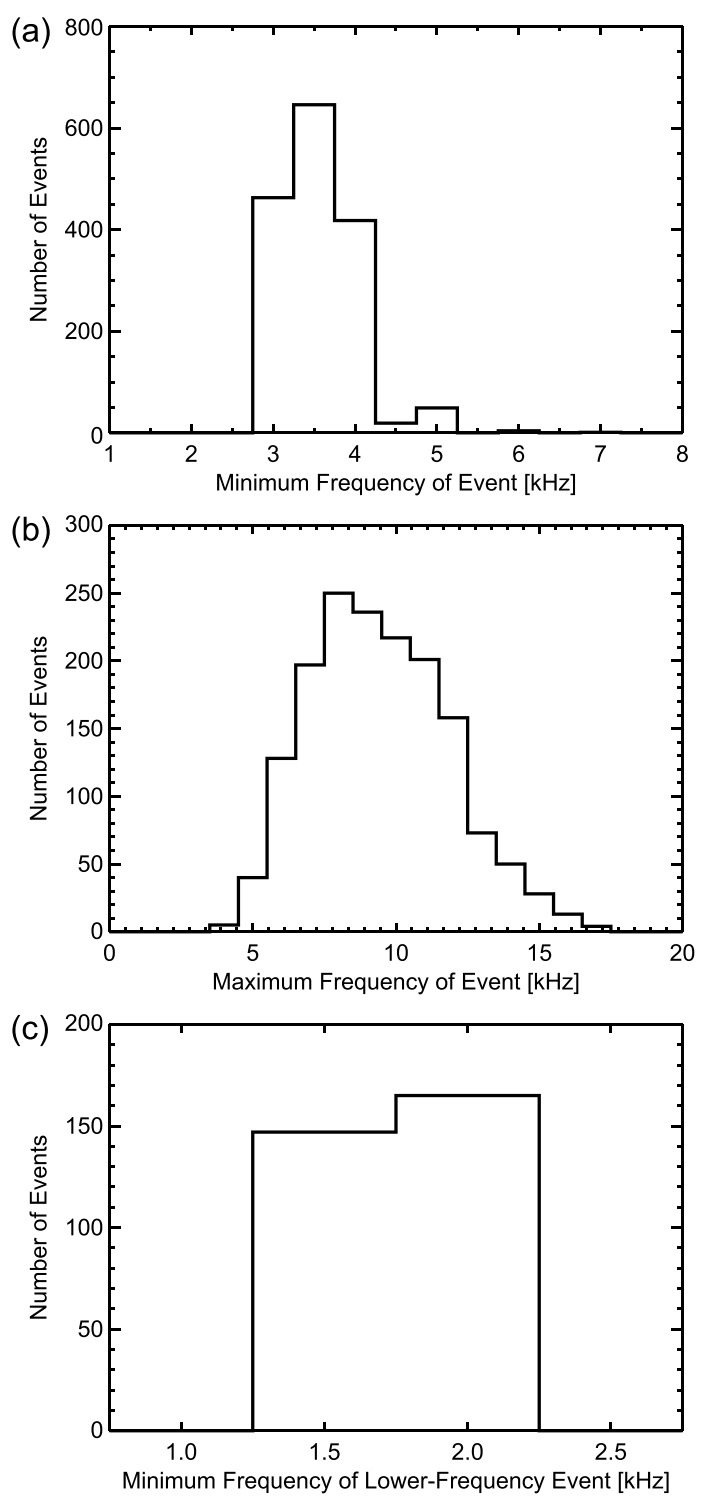

Figure 5. (a) Histogram of minimum frequencies of events. (b) Histogram of maximum frequencies of events. (c) Histogram of minimum frequencies of lower frequency events. The frequency resolution of all these histograms is $0.5 \mathrm{kHz}$.

tive to compare their global occurrence with the long-term average lightning activity from LIS/OTD. For this purpose, the average lightning activity in the local time interval 20.7-23.7 $\mathrm{h}$ (i.e., the local time interval corresponding to the DEMETER nightside measurements) is plotted in Figure $6 \mathrm{~b}$. The average number of lightning strokes per square kilometer per year in each $2.5^{\circ} \times 2.5^{\circ}$ bin is color coded according to the scale on the top.

One can identify three main areas of an increased lightning activity. These are located in central Africa and in both North and South America. We note that the lightning distribution varies during the year, with lightning activity being enhanced during the months corresponding to the local summer (depending on the hemisphere). However, for our purpose the overall average lightning activity is sufficient. Comparing Figures $6 a$ and $6 \mathrm{~b}$, one can see that the three areas of increased occurrence of the reduced intensity events appear to be related to the three areas of increased lightning activity, but they are shifted in the western direction. Moreover, the two areas of increased occurrence of the reduced intensity events close to America appear to be shifted also in the southern direction.

The fact that the reduced intensity events do not occur at locations of the highest lightning activity but they are somewhat shifted can be likely explained by a hypothesis that the lightning-generated emissions need to 
(a)
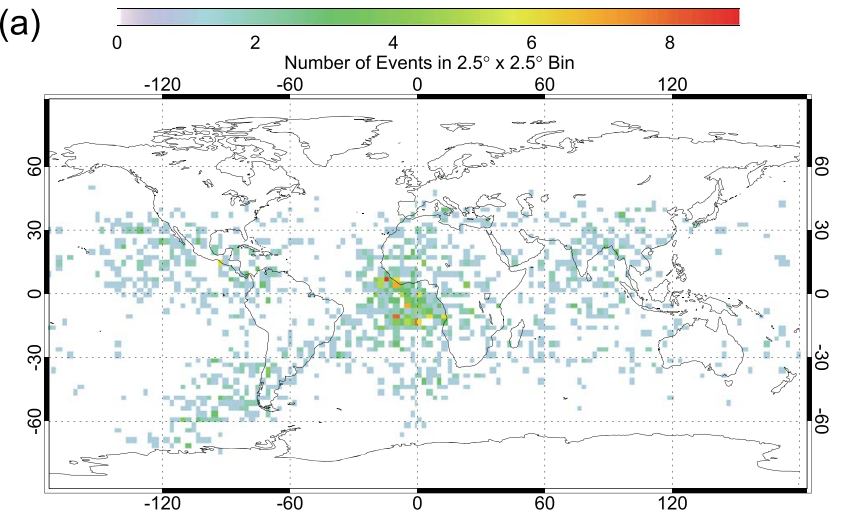

(b)

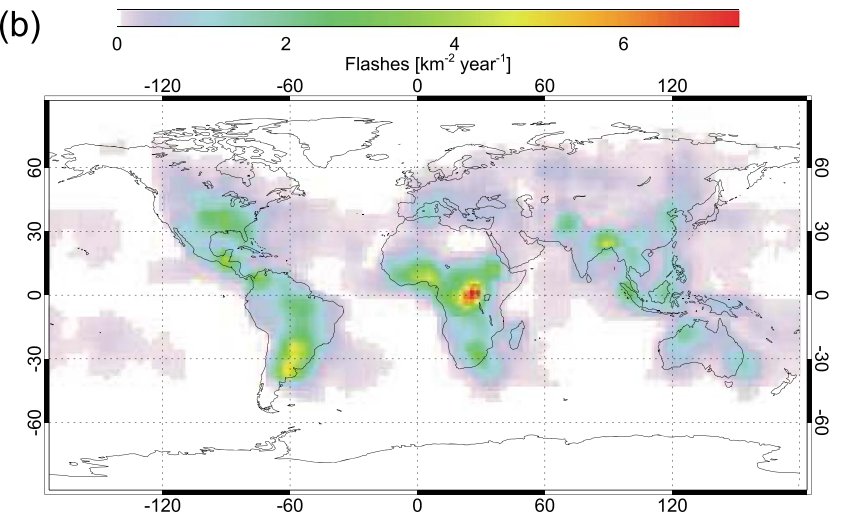

Figure 6. (a) Map of event locations. The location of a given event is determined as the location of the DEMETER spacecraft at the time of the minimum of the event frequency dependence. The number of events in each $2.5^{\circ} \times 2.5^{\circ}$ bins is color coded according to the scale on the top. (b) The average lightning activity from LIS/OTD in the local time window $20.7-23.7 \mathrm{~h}$ is color coded according to the scale at the top. This local time range corresponds to DEMETER nighttime half orbits.

propagate some distance in the Earth-ionosphere waveguide to form the reduced intensity events. However, in order to try to understand the positional shift of the reduced intensity events with respect to the lightning locations, one needs to take into account the orbit of the DEMETER spacecraft. This orbit is nearly polar and quasi Sun-synchronous, with the satellite moving from the south to the north and slightly to the west on the nightside. The shape of a DEMETER nightside half orbit is shown in Figure 7a. It was moved arbitrarily in the east-west direction in order to go through the longitude equal to 0 at the equator. Hypothesizing that
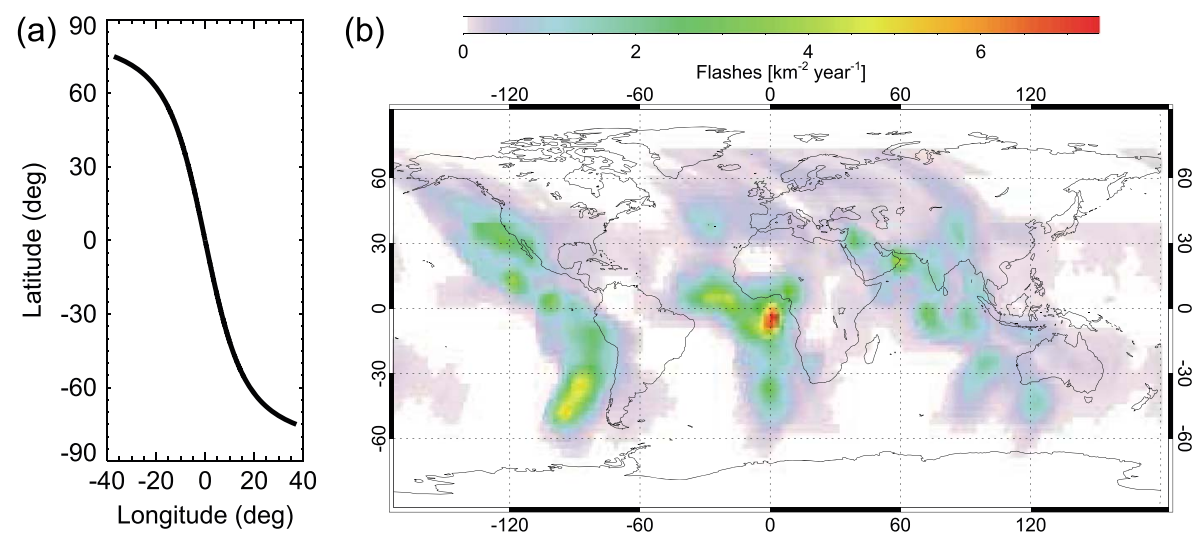

Figure 7. (a) Trajectory corresponding to a DEMETER nightside half orbit. (b) Map of lightning locations from LIS/OTD shifted by $3000 \mathrm{~km}$ in the west/southwest direction. The direction of the shift was determined in each location to be perpendicular to the DEMETER orbit at the resulting shifted location; i.e., the shifted location is the place where the DEMETER spacecraft gets closest to the original location. 
the locations of reduced intensity events plotted in Figure 6a correspond to the positions when the spacecraft was closest to the source storms, and that the reduced intensity events are typically formed at some characteristic distance from the source storm, this analysis can help us to at least partly understand the positional shift of the reduced intensity events.

When one assumes that the position of a reduced intensity event is defined by its frequency minimum and that the distance between this position and a source storm would be the lowest all over a given DEMETER half orbit, it is clear that the line connecting the location of the reduced intensity event and the source storm must be perpendicular to the appropriate DEMETER half orbit at the location of the reduced intensity event. Taking into account the shape of DEMETER nightside half orbits and hypothesizing further that the events are formed preferentially at some characteristic distance from the source storm, this necessarily results in reduced intensity events formed either to the west/southwest from source storms or in reduced intensity events formed to the east/northeast from source storms. Nevertheless, we can only guess why the reduced intensity events are formed preferentially in the west/southwest direction (see section 4).

As for the characteristic distance between reduced intensity events and source storms, we can perform the analysis shown in Figure 7b. Assuming some value of the characteristic distance, we shift the lightning activity bins from Figure $6 \mathrm{~b}$ by this distance in the direction perpendicular to the DEMETER orbit at the resulting shifted location. Out of the two possible solutions which fulfill this condition, we choose the shift in the west/southwest direction, corresponding to the observed situation. The resulting shifted map of the average lightning activity from LIS/OTD is then compared with the map of the event occurrence from Figure 6a. This allows us to determine such a characteristic distance between reduced intensity events and source storms which results in the best visual agreement between the two maps. It is found that this best agreement characteristic distance is equal to about $3000 \mathrm{~km}$. The resulting map of lightning activity shifted using the above described approach by $3000 \mathrm{~km}$ is shown in Figure 7b. A direct comparison of this shifted lightning map with the map of the event occurrence from Figure $6 a$ shows a reasonable agreement. This means that the overall geographic distribution of the events is shifted by a characteristic distance and in a specific direction reflecting the DEMETER orbit with respect to the global lightning activity. Although this reveals a typical picture averaged over all the events, it is important to note that individual events may behave rather differently (see, e.g., the example event in Figure 8).

We have suggested that the formation of the reduced intensity events is related to the propagation of lightning-generated waves in the Earth-ionosphere waveguide prior to their leakage to the DEMETER altitudes. In order to verify and demonstrate this idea in more detail, we selected two well-pronounced reduced intensity events for a detailed analysis. The frequency-time spectrograms of power spectral density of electric field fluctuations corresponding to these events are shown in Figures $8 c$ and $9 c$, respectively. The first event was measured on 29 March 2010, and the second event was measured on 22 September 2007. The black horizontal lines in the figures show the frequencies of $1.7 \mathrm{kHz}$ and $3.4 \mathrm{kHz}$, respectively, i.e., the frequencies corresponding to typical nightside waveguide critical frequency and its double. The black vertical lines in the figures mark the times when DEMETER gets closest to the source storms (see below).

The corresponding satellite orbits are shown in Figures $8 \mathrm{a}$ and $9 \mathrm{a}$ by the thin curves. The thick curves correspond to the parts of the orbits when the events were observed (i.e., to the time intervals plotted in the frequency-time spectrograms). We have used the WWLLN data to investigate the lightning activity during these time intervals. The numbers of lightning strokes detected by WWLLN during the event time intervals in $5^{\circ} \times 5^{\circ}$ bins are color coded according to the scales at the top.

The thin horizontal and vertical lines mark the positions of source storms. These were determined using Figures $8 \mathrm{~b}$ and $9 \mathrm{~b}$. In these figures, the distances between individual lightning strokes detected by WWLLN and the DEMETER spacecraft are shown by the black points as a function of time. If a single dominating localized storm was present during the event occurrence, the individual points would lie close to a curve corresponding to the spacecraft movement with respect to the storm center. This is indeed the case in Figure $8 \mathrm{~b}$. The appropriate distance-time curve calculated assuming a source storm located at the intersection of the thin horizontal and vertical lines in Figure $8 a$ is shown by the red curve. It can be seen that a substantial number of black points is well fitted by this curve, indicating that the assumption of a dominating localized storm is reasonable. 
(a)
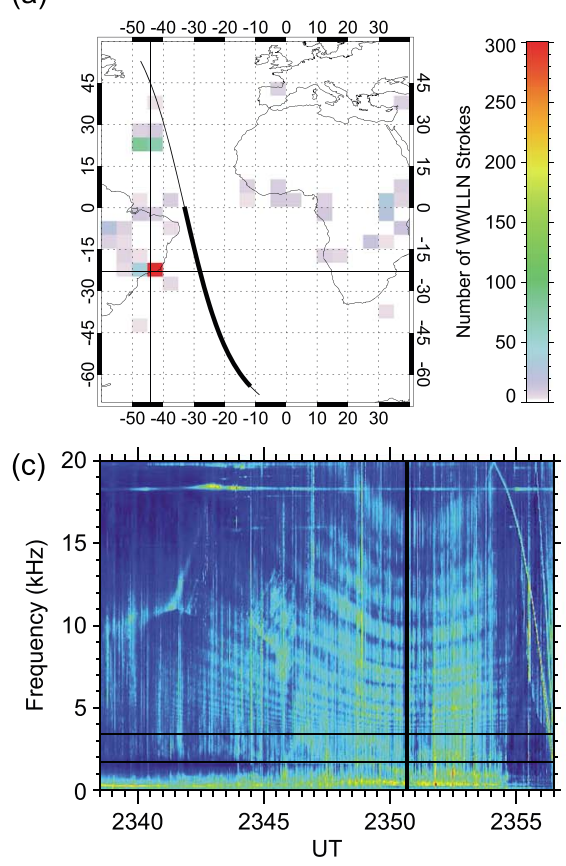

(b)
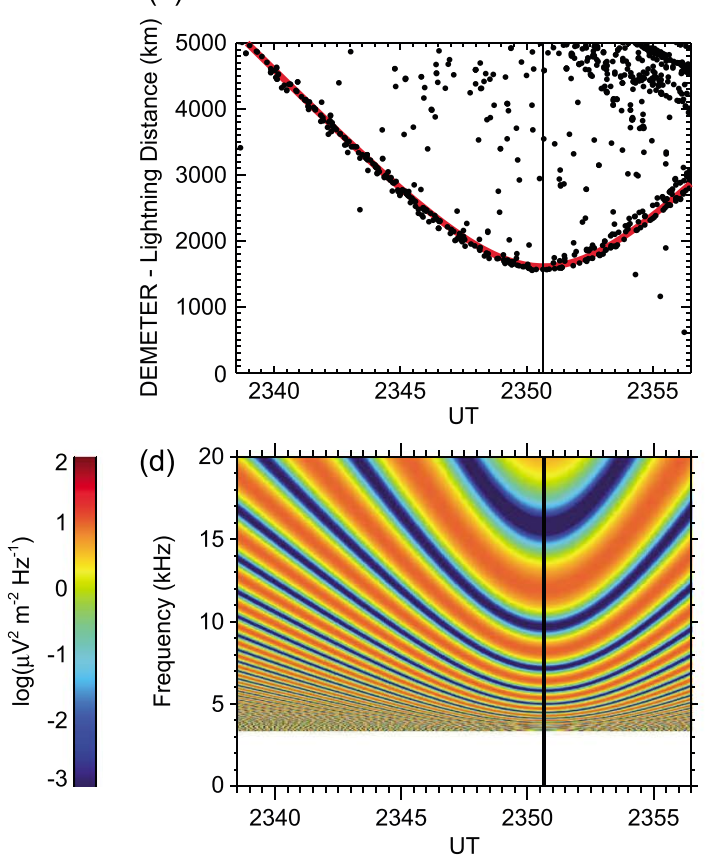

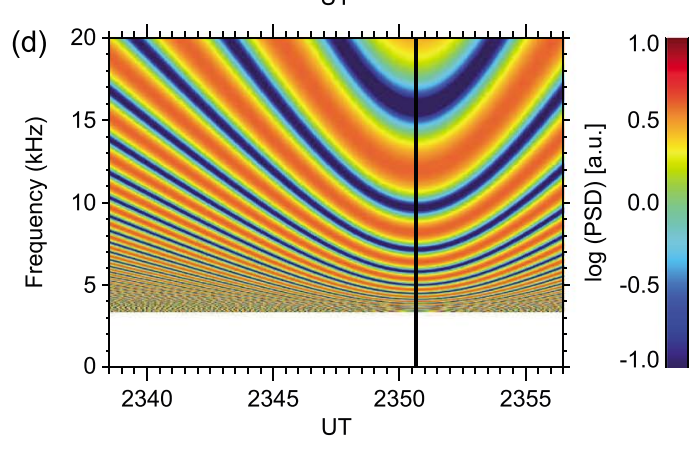

Figure 8. (a) DEMETER orbit is shown by the thin curve. The thick curve corresponds to the part of the DEMETER orbit where the event was observed. Numbers of lightning strokes detected during this time interval by the WWLLN network in individual $5^{\circ} \times 5^{\circ}$ bins are color coded according to the scale at the top. The thin horizontal and vertical lines mark the position of a source storm (see text). (b) Distances between individual lightning strokes detected by WWLLN and the DEMETER spacecraft are shown by the black points as a function of time. The red curve shows the distance between the DEMETER spacecraft and the location of the source storm. The black vertical line marks the time when DEMETER gets closest to the source storm. (c) Frequency-time spectrogram of power spectral density of electric field fluctuations corresponding to the event. The data were measured on 29 March 2010. (d) Model frequency-time spectrogram calculated using modal interference between TM1 and TM2 modes of the Earth-ionosphere waveguide.

The situation in Figure $9 \mathrm{~b}$ is somewhat less clear. It is more complex, with several different storms possibly playing a role. Nevertheless, a significant number of lightning strokes lie close to the red curve, indicating that one of the storms can be probably again considered as dominant. The black vertical line marks the time when DEMETER got closest to the source storm. It is noteworthy that the minimum distance between the spacecraft and the source storm is significantly lower in this event than it was in the event from Figure 8. It is also noteworthy that the event from Figure 8 occurred to the east from the source storm, as the events typically tend to occur to the west (see above). Finally, we note that while the used approach allows us to determine the source location in clear cases (Figure 8), it struggles with more complex events (Figure 9) and it ultimately fails at the times of high lightning activity taking place simultaneously at several locations. This is why it can be used for case studies, but it cannot provide us with the overall distribution of source storm locations.

Having identified the locations of the source storms, we can try, following a model described by Parrot et al. [2008], to develop a simple model of the event formation using an idealized waveguide mode theory. We consider a planar Earth surface and a planar lower boundary of the ionosphere, and we assume that both are perfectly conducting. Using the coordinate system where the Earth surface lies in the $z=0$ plane and the bottom of the ionosphere lies in the $z=h$ plane, the electric field intensity $E$ of a signal with the frequency $f$ and the wave vector $k=2 \pi f / c$ ( $c$ is the speed of light) at a horizontal distance $x$ from the source is then [Budden, 1961]

$$
E(f, x) \propto \exp (-i k x \cos \theta)
$$

where $\theta$ is the angle between the wave normal and the horizontal direction. It is, for a given waveguide mode number $n$, given by 
(a)

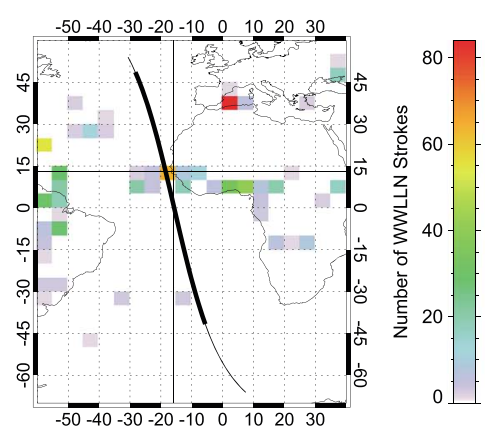

(c)

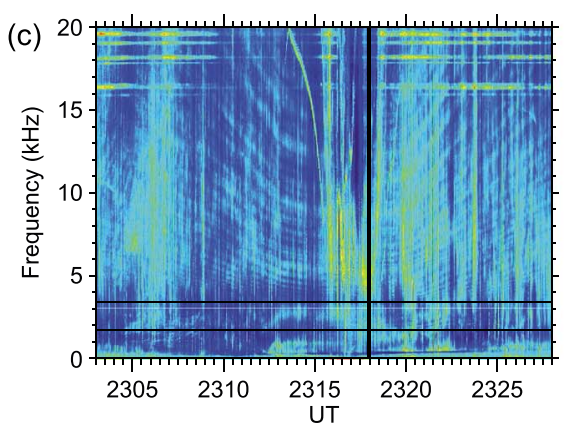

(b)
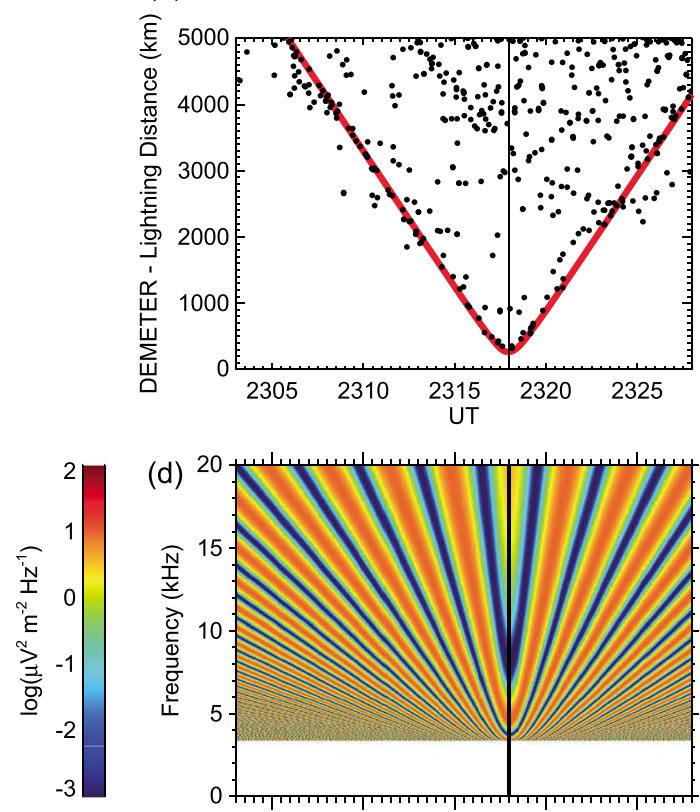

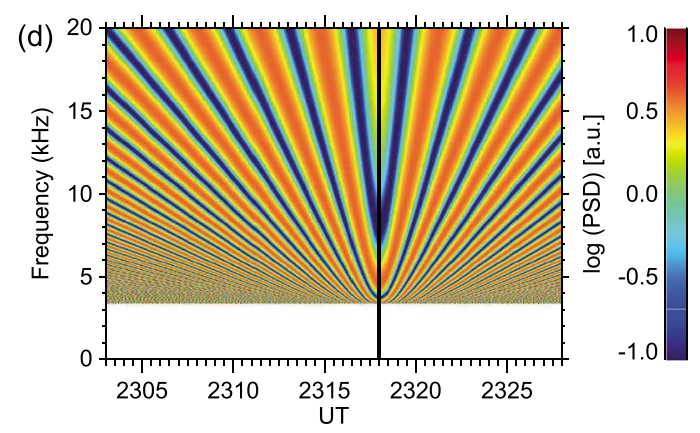

Figure 9. The same as Figure 8 but for the event measured on 22 September 2007. Note that the situation is less clear than in Figure 8, with several different storms occurring in the vicinity of the DEMETER spacecraft at the time of the event. Also note that the DEMETER spacecraft passed very close to the source storm in this particular event.

We note that for a given waveguide mode number, only frequencies higher than the critical frequency

$$
f_{n}=\frac{n c}{2 h}
$$

can propagate in the waveguide.

For the purpose of our simple model calculation, we adopt a typical value of the nighttime waveguide critical frequency $f_{1}=1.7 \mathrm{kHz}$. This corresponds to the effective waveguide height $h \approx 88 \mathrm{~km}$, and it is well consistent with the investigated frequency-time spectrograms (see the intensity cutoffs close to the lower horizontal black lines marking $f_{1}=1.7 \mathrm{kHz}$ in Figures $8 \mathrm{c}$ and $9 \mathrm{c}$ ). The effective height of the waveguide may vary over the propagation path. This might be especially important close to the terminator where the changes are expected to be substantial. However, in our simple model we consider the effective waveguide height to be constant.

Taking into account that higher-order modes are attenuated significantly more than lower order modes [e.g., Wait, 1962] and following Parrot et al. [2008], we limit, in the first approximation, our calculation only to the first two modes (TM1 and TM2). The total intensity of a signal at a distance $x$ from the source is then calculated as a sum of these two modes. The intensities of these two modes are described by complex dependencies given by equations ( 1 ) and (2), considering $n=1$ and $n=2$, respectively. The two modes interfere, and the resulting intensity of the signal at a given frequency changes periodically with the distance from the source. Moreover, the resulting signal intensity at a given distance from the source changes periodically with the frequency of the signal. It is clear that in our simplified model, this modulation occurs only at frequencies higher than the critical frequency of the TM2 mode (equation (3) for $n=2$ ), i.e., at frequencies where both TM1 and TM2 modes can propagate. In order to get the interference below the critical frequency of the TM2 mode and explain occasionally observed lower frequency reduced intensity events, one would have to consider the TM0 mode and the interference between TM0 and TM1 modes in our model.

We use this very simplified model of the modal interference in the Earth-ionosphere waveguide to calculate theoretical frequency-time spectrograms corresponding to the two selected reduced intensity events. The horizontal distance $x$ of the signal propagation in the Earth-ionosphere waveguide is considered to be equal to the distance between the source storm and the satellite projection on the ground. The intensities of TM1 and TM2 modes are assumed to be the same. The obtained results are shown in Figures $8 \mathrm{~d}$ and $9 \mathrm{~d}$. It can 
be seen that the resulting modeled frequency-time spectrograms are comparable to the shape of the measured spectrograms. The modeled patterns of frequencies with a reduced intensity have a minimum at the time when DEMETER gets closest to the source storm (marked by the black vertical line). Moreover, the modeled variation of the frequencies with a reduced intensity is comparable with the observed situation. Finally, the frequency separation between individual frequencies of reduced intensity increases as a function of frequency, again in agreement with the observations. It is, however, clear that the exact structure of the reduced intensity events cannot be properly reproduced by our simple model. This can be particularly well seen at higher frequencies, where more frequencies of reduced intensity are observed than predicted by the model. Moreover, the data-model agreement for the event analyzed in Figure 9 is significantly worse than that for the event analyzed in Figure 8, particularly at the times when the DEMETER spacecraft was close to the source storm. This will be discussed more in detail in section 4.

\section{Discussion}

Detailed frequency-time spectrograms available during the times of the active Burst mode reveal that the reduced intensity events are composed of individual lightning-generated $0+$ whistlers, whose intensity at some specific frequencies is significantly attenuated. This attenuation, which is related to the propagation in the Earth-ionosphere waveguide, is in principle present any time DEMETER detects lightning-generated whistlers which experienced the propagation in the Earth-ionosphere waveguide before penetrating the ionosphere from below. However, in order to form a detectable reduced intensity event, the whistler occurrence rate must be high enough to result in apparently nearly continuous smooth reduced intensity curves in the Survey mode data with the time resolution of $2 \mathrm{~s}$.

The reduced intensity events were visually identified in frequency-time spectrograms of power spectral density of electric field fluctuations. This visual identification is necessarily partly subjective. Most importantly, only events which are sufficiently clear and which last a sufficiently long time were identified. The event thus has to last sufficiently long (on the order of minutes) and consist of a high number of individual whistlers (several per second) in order to be properly identified. This inevitably limits the used data set to only the best pronounced events. It is also likely the reason why no events with a very short duration were identified. However, the events were systematically identified in all data measured by the DEMETER spacecraft, and the - albeit sometimes rather subjective - criteria of the event identification remained the same. Consequently, we believe that there is no sampling bias in the sense that there is no reason why the resulting set of identified events should prefer some specific times and/or locations.

The suggested model of the event formation based on the modal interference in the Earth-ionosphere waveguide [Parrot et al., 2008] explains well some basic event properties. Considering that at least two wave modes have to be able to propagate at a given frequency in order to interfere, one can directly explain the observed cutoff frequencies. Taking into account that the typical value of the waveguide critical frequency during the night is about $1.7 \mathrm{kHz}$, the lower frequency cutoff of the reduced intensity events at about $3.4 \mathrm{kHz}$ is due to the fact that the TM2 mode necessary for the modal interference can propagate only above this frequency. The only exception might be the modal interference between TM0 and TM1 modes, which is likely responsible for the formation of the lower frequency reduced intensity events. These are, however, typically very weak, and they are observed only for comparatively short times. We note that the accuracy of the cutoff determination from the experimental data is not very high $(0.5 \mathrm{kHz})$. However, the predicted cutoff frequencies are consistent with the observations. Another feature of the reduced intensity events explainable by the simple modal interference model is the fact that the frequency separation of the reduced intensity frequencies is larger at higher frequencies. The analysis of two selected reduced intensity events reveals that the predicted frequency slope of the reduced intensity curves - related to the varying distance between the satellite and the source storm - is in a reasonable agreement with the observations as well.

The formation of the reduced intensity events and their basic properties thus seem to be explainable by the modal interference. There are, however, two important points that this simple model is not able to explain properly. First, it is the exact frequency structure of the events. Particularly at higher frequencies the observed frequencies of reduced intensity do not correspond to the predicted ones, and there is, in general, a trend of more frequencies with a reduced intensity observed than predicted. This is likely related to the fact that while our simplified calculation considers only the first two waveguide modes, there are additional modes occurring at higher frequencies. These higher modes inevitably result to additional interference frequencies and 
thus additional frequencies with a reduced intensity in the frequency spectra. However, the exact consideration of these higher-order modes is complicated, as it would require the proper evaluation of their intensities; i.e., one would have to consider both the attenuation of individual modes during their propagation in the Earth-ionosphere waveguide and their relative excitation by the source lightning. Moreover, one should also consider that due to the not entirely sharp lower boundary of the ionosphere, the cutoff frequencies of individual waveguide modes are not exactly harmonic [Kumar et al., 2008]. A proper full wave analysis of another similar event has been done by Parrot et al. [2008].

Another important observational fact which is not explainable by the simple modal interference model is the positional shift of the observed events with respect to the probable source lightning locations. Specifically, although the observed west/southwest shift has been shown to be related to the spacecraft orbit, it remains unexplained why it is so much preferred as compared to the east/northeast shift and why the events are formed preferentially at distances of about $3000 \mathrm{~km}$ from source lightning locations. In this regard, one should consider whether the average global lightning occurrence map used to determine the positional shift is optimal, as it does not take into account the properties of individual lightning nor their instantaneous occurrence rate, only the long-term average. These can be, however, significantly different at different locations. It is, for example, known that lightning observed above oceans are generally stronger and last longer than lightning observed above land [Beirle et al., 2014]. On the other hand, land thunderstorms typically have higher flash rates [Ushio et al., 2001]. Assuming that the thunderstorms/lightning with some specific properties are more likely to generate the reduced intensity events and assuming that they are located at some specific regions, a comparison of the event locations with the average lightning map may not be sufficient. However, a comparison of the shifted average lightning map with the event locations shows a surprisingly good agreement, indicating that the event observations to the west/southwest from the areas of enhanced average lightning occurrence are likely really due to emissions propagating from these areas.

As for the explanation of the optimal positional shift direction and distance, we can only speculate. It seems reasonable that the lightning-generated emissions have to propagate in the Earth-ionosphere waveguide for some distance so that there is a phase shift between individual waveguide modes, allowing for the negative interference at some frequencies. Moreover, at larger distances higher-order waveguide modes get attenuated, simplifying thus the resulting interference pattern. The experimentally estimated optimum distance of about $3000 \mathrm{~km}$ for the event occurrence might be a compromise between these effects and the overall attenuation of the lightning-generated emissions with distance, which prevents the event formation at too large distances from source storms. The low intensity of $0+$ whistlers detected by the DEMETER spacecraft during the daytime caused by the large ionospheric attenuation [e.g., Němec et al., 2008] is also likely the reason why reduced intensity events are observed exclusively during the night.

Concerning the question why the west/southwest shift of the events with respect to the storm locations is preferred as compared to the east/northeast shift, the situation is even more complicated. There is an attenuation difference between the eastward and westward propagation in the Earth-ionosphere waveguide. This asymmetry is introduced by the Earth's magnetic field, which results in the azimuthally dependent attenuation. The waves exhibit larger attenuation when propagating westward than when propagating eastward [Crombie, 1958; Barber and Crombie, 1959; Dobrott and Ishimaru, 1961; Budden, 1968; Jacobson et al., 2012; Burkholder et al., 2013]. Recent experimental results obtained using the WWLLN data showed that the average daytime attenuation of spherics in the $8-18 \mathrm{kHz}$ band propagating in the Earth-ionosphere waveguide over the water is $1.13 \pm 0.35 \mathrm{~dB} / \mathrm{Mm}$ and $2.98 \pm 0.68 \mathrm{~dB} / \mathrm{Mm}$ for eastward propagating and westward propagating spherics, respectively [Hutchins et al., 2013]. These values are well comparable with former experimentally determined attenuation rates [Taylor, 1960]. During the nighttime, the average attenuation rates are $0.71 \pm 0.68 \mathrm{~dB} / \mathrm{Mm}$ and $2.66 \pm 0.39 \mathrm{~dB} / \mathrm{Mm}$ for eastward and westward propagation, respectively [Hutchins et al., 2013].

It is, however, not clear how exactly this asymmetric attenuation in the waveguide should result in the asymmetric shift of the event locations. Roughly speaking, it might be possible that for a reduced intensity event to occur, a single localized storm must be a dominant source of emissions detected by the spacecraft. If the spacecraft is located to the east of the storm centers, this might be difficult to achieve, as lightning originating at several different storms are detected, at the same time, due to the low attenuation in the waveguide. On the other hand, if the spacecraft is located to the west of the storm centers, a single-source storm might possibly dominate, as the lightning originating at other, more distant, storms are sufficiently attenuated 
not to play a role in the event formation. Let us assume two storms with the same flash rate and lightning intensity. Considering the fixed scale of frequency-time spectrograms used for the identification of events $\left(10^{-3}\right.$ to $\left.10^{2} \mu \mathrm{V}^{2} \mathrm{~m}^{-2} \mathrm{~Hz}^{-1}\right)$, the power spectral density of resulting whistlers should differ by about $5 \mathrm{~dB}$ for the whistlers from one storm to be sufficiently dominant. Taking into account the average nighttime attenuation rates reported by Hutchins et al. [2013], the storm-spacecraft distances would have to differ by about $7000 \mathrm{~km}$ for the waves propagating eastward but by less than $2000 \mathrm{~km}$ for the waves propagating westward. The event from Figure 8 might be well consistent with this picture. Although it occurred to the east from the source storm, other storms at that time were clearly too weak and distant to affect the situation.

Another possibility to explain the westward rather than eastward shift of the event locations with respect to the lightning locations might be to consider the terminator effects on the Earth-ionosphere waveguide propagation. DEMETER nighttime observations take place around 22:30 LT. The average spatial separation of the observed events from the terminator therefore approximately is $7500 \mathrm{~km}$. Taking into account the spatial separation of the observed events from the source storms of about $3000 \mathrm{~km}$, the propagation path is generally entirely nighttime, with likely quite uniform Earth-ionosphere waveguide. Thus, this excludes the influence of the terminator effects.

\section{Conclusions}

We demonstrated the existence of unusual events with a reduced intensity present in the electromagnetic wave data measured by the DEMETER spacecraft in the VLF range. It was demonstrated that these events are formed by $0+$ whistlers whose intensity is significantly attenuated at specific frequencies. When the whistler occurrence rate is high enough, the smooth variation of the frequencies with a reduced intensity results in distinct curves of reduced intensity which are identifiable in frequency-time spectrograms. Such reduced intensity events were identified by a systematic inspection of all available DEMETER data, resulting in a data set of 1601 events. About $20 \%$ of cases are accompanied by additional reduced intensity events observed at lower frequencies. The events occur exclusively during the night.

We have suggested a simple model of the event formation based on modal interference of lightning-generated electromagnetic waves propagating in the Earth-ionosphere waveguide, and we applied this model to two selected events. The model is able to reproduce some of the observed event characteristics (minimum cutoff frequencies and approximate frequency-time shape of events).

The overall geographic distribution of the events is shifted by about $3000 \mathrm{~km}$ westward and slightly southward with respect to the areas with high long-term average lightning activity. We demonstrated that this shift is related to the specific DEMETER orbit. The optimum distance of the shift is likely a compromise between the necessity to attenuate higher-order waveguide modes and the overall attenuation of the emissions with distance. The west/southwest shift might be preferred as compared to the east/northeast shift due to the azimuthal asymmetry of the waveguide attenuation.

Lightning-generated whistlers thus routinely penetrate through the ionosphere at distances as large as a few thousands of kilometers from the source storm. This is a much larger region than is typically assumed when considering the effect of a single thunderstorm on the ionosphere and magnetosphere. Due to the propagation in the Earth-ionosphere waveguide, the frequency spectrum of a series of whistlers coming from an isolated thunderstorm sometimes shows a characteristic modulation which can be explained by the interference of different waveguide modes of propagating signals. This specific pattern of the whistler frequency spectrum should be considered when evaluating the effects of lightning on the Earth's electromagnetic environment and/or energetic particles.

\section{References}

Barber, N. F., and D. D. Crombie (1959), V.L.F. reflections from the ionosphere in the presence of a transverse magnetic field, J. Atmos. Terr. Phys, 16(1-2), 37-45.

Beirle, S., W. Koshak, R. Blakeslee, and T. Wagner (2014), Global patterns of lightning properties derived by OTD and LIS, Nat. Hazards Earth Syst. Sci., 14, 2715-2726, doi:10.5194/nhess-14-2715-2014.

Berthelier, J. J., et al. (2006), ICE, the electric field experiment on DEMETER, Planet. Space Sci., 54, 456-471.

Budden, K. G. (1961), The Wave-Guide Mode Theory of Wave Propagation, Logos Press, London.

Budden, K. G. (1968), The Propagation of Radio Waves, Cambridge Univ. Press, Cambridge, U. K.

Burkholder, B. S., M. L. Hutchins, M. P. McCarhy, R. F. Pfaff, and R. H. Holzworth (2013), Attenuation of lightning-produced sferics in the Earth-ionosphere waveguide and low-latitude ionosphere, J. Geophys. Res. Space Physics, 118, 3692-3699, doi:10.1002/jgra.50351. 
Christian, H. J., et al. (2003), Global frequency and distribution of lightning as observed from space by the Optical Transient Detector, J. Geophys. Res., 108(D1), 4005, doi:10.1029/2002JD002347.

Crombie, D. D. (1958), Differences between the east-west and west-east propagation of VLF signals over long distances, J. Atmos. Terr. Phys., $12(2-3), 110-117$.

Cummer, S. A. (2000), Modeling electromagnetic propagation in the Earth-ionosphere waveguide, IEEE Trans. Antennas Propag., 48(9), $1420-1429$.

Dobrott, D., and A. Ishimaru (1961), East-west effect on VLF mode transmission across the Earth's magnetic field, J. Res. Nat. Bur. Stand., $65 D(1), 47-52$.

Helliwell, R. A. (1965), Whistlers and Related lonospheric Phenomena, Stanford Univ. Press, Stanford, Calif.

Hutchins, M. L., A. R. Jacobson, R. H. Holzworth, and J. B. Brundell (2013), Azimuthal dependence of VLF propagation, J. Geophys. Res. Space Physics, 118, 5808-5812, doi:10.1002/jgra.50533.

Jacobson, A. R., X.-M. Shao, and E. Lay (2012), Time domain waveform and azimuth variation of ionospherically reflected VLF/LF radio emissions from lightning, Radio Sci., 47, RS4001, doi:10.1029/2012RS004980.

Kumar, S., A. Kishore, and V. Ramachandran (2008), Higher harmonic tweek sferics observed at low latitude: Estimation of VLF reflection heights and tweek propagation distance, Ann. Geophys., 26, 1451-1459, doi:10.5194/angeo-26-1451-2008.

Lay, E. H., R. H. Holzworth, C. J. Rodger, J. N. Thomas, O. Pinto Jr., and R. L. Dowden (2004), WWLL global lightning detection system: Regional validation study in Brazil, Geophys. Res. Lett., 31, L03102, doi:10.1029/2003GL018882.

Mazouz, F. E.-L., J.-L. Pincon, M. Parrot, H. D. Feraudy, N. G. Lehtinen, and F. Lefeuvre (2011), Asymmetric V-shaped streaks recorded on board DEMETER satellite above powerful thunderstorms, J. Geophys. Res., 116, A11321, doi:10.1029/2011JA016794.

Němec, F., O. Santolík, M. Parrot, and J. Bortnik (2008), Power line harmonic radiation observed by satellite: Properties and propagation through the ionosphere, J. Geophys. Res., 113, A08317, doi:10.1029/2008JA013184.

Němec, F., O. Santolík, M. Parrot, and C. J. Rodger (2010), Relationship between median intensities of electromagnetic emissions in the VLF range and lightning activity, J. Geophys. Res., 115, A08315, doi:10.1029/2010JA015296.

Parrot, M., et al. (2006), The magnetic field experiment IMSC and its data processing onboard DEMETER: Scientific objectives, description and first results, Planet. Space Sci., 54, 441-455.

Parrot, M., U. S. Inan, and N. G. Lehtinen (2008), V-shaped VLF streaks recorded on DEMETER above powerful thunderstorms, J. Geophys. Res., 113, A10310, doi:10.1029/2008JA013336.

Parrot, M., et al. (2015), Unexpected very low frequency (VLF) radio events recorded by the ionospheric satellite DEMETER, Surv. Geophys., 36, 483-511, doi:10.1007/s10712-015-9315-5.

Rodger, C. J., J. B. Brundell, R. L. Dowden, and N. R. Thomson (2004), Location and accuracy of long distance VLF lightning location network, Ann. Geophys., 22, 747-758.

Taylor, W. L. (1960), VLF attenuation for east-west and west-east daytime propagation using atmospherics, J. Geophys. Res., 65(7), 1933-1938.

Toledo-Redondo, S., M. Parrot, and A. Salinas (2012), Variation of the first cut-off frequency of the Earth-ionosphere waveguide observed by DEMETER, J. Geophys. Res., 117, A04321, doi:10.1029/2011JA017400.

Ushio, T., S. J. Heckman, D. J. Boccippio, H. J. Christian, and Z.-I. Kawasaki (2001), A survey of thunderstorm flash rates compared to cloud top height using TRMM satellite data, J. Geophys. Res., 106(D20), 24,089-24,095.

Wait, J. R. (1962), Introduction to the theory of VLF propagation, Proc. IRE, 50(7), 1624-1647. 